\title{
Blood Pressure Response to Exercise in Unaffected Relatives of Polycystic Kidney Disease Patients
}

\author{
Ezgi Yenigun Coskun ${ }^{1}$, Didem Turgut ${ }^{2}$, Simal Koksal Cevher ${ }^{1}$, Cigdem Yucel ${ }^{3}$, Cenk \\ Aypak $^{4}$, and Fatih Dede ${ }^{1}$ \\ ${ }^{1}$ The University of Health Sciences, Ankara City Hospital \\ ${ }^{2}$ Baskent University Ankara Hospital \\ ${ }^{3}$ The University of Health Science, Ankara Gulhane Education and Research Hospital \\ ${ }^{4}$ The University of Health Sciences, Diskapi Yildirim Education and Research Hospital
}

February 14, 2021

\begin{abstract}
Background Hypertension is an early finding of autosomal dominant polycystic kidney disease (ADPKD) and is related to different mechanisms. Cyst expansion related renin secretion or early endothelial dysfunctions are some of these hypotheses. Different course of hypertension in ADPKD preoccupies that relatives of ADPKD patients may also be under risk for this underlying mechanisms. In this study, we aimed to find blood pressure response problems to exercise as an initial vascular problem in unaffected relatives of hypertensive ADPKD patients. Methods The cross-sectional study included 24 unaffected relatives (siblings and children) of ADPKD patients and 30 healthy controls that performed a cycle ergometer test. Additionally, as a marker for endothelial function, nitric oxide (NO) and asymmetric dimethylarginine (ADMA) levels at baseline and post exercise were measured. Results Systolic blood pressure (SBP) and diastolic blood pressure (DBP) increases were similar in both groups during the 1st, 3rd, and 6th min of exercise. During the exercise recovery phase SBP decreased in both groups; however, in the relatives of ADPKD patients DBP remained high at the end of the 6th min, suggesting impaired capacity for exercise-induced vasodilatation. Baseline NO and ADMA, and 1-min NO and ADMA were similar in both groups. Conclusion Abnormal blood pressure response to exercise stress was observed in unaffected normotensive relatives. The observed abnormal DBP response pattern to exercise suggests that arterial vascular responses might be already altered in unaffected relatives of hypertensive ADPKD patients; however, long-term clinical trials are needed to clarify the significance of these findings.
\end{abstract}

\section{Hosted file}

Main Text.pdf available at https://authorea.com/users/395724/articles/508868-blood-pressureresponse-to-exercise-in-unaffected-relatives-of-polycystic-kidney-disease-patients

\section{Hosted file}

tables.pdf available at https://authorea.com/users/395724/articles/508868-blood-pressureresponse-to-exercise-in-unaffected-relatives-of-polycystic-kidney-disease-patients 